\title{
Social Semiotics and the Related Interpretation
}

\author{
Jinshun Long ${ }^{1 * a}$, Jun $\mathrm{He}^{2 \mathrm{~b}}$ \\ ${ }^{1}$ School of Foreign Languages, Jimei University, Xiamen, Fujian, China \\ ${ }^{2}$ School of Architecture and Civil Engineering, Jinggangshang University, Ji'an, Jiangxi, China \\ a*Jinshun Long. jslong123@sina.com
}

\begin{abstract}
Social semiotics studies cultural and community-specific semiotic practices in order to produce various texts and meanings in the contexts of various situations and of culturally meaningful activities. From the perspective of social semiotics, our understanding of reality cannot be separated from the actual compiled code and semantic system, which means that social (cultural) reality itself is a building full of meanings----a kind of semiotic structure. For this reason, text, style, code, language system and its social structure are put into the theoretical study of semiotics. Thus social semiotics is to study the social dimension of meaning, and of the power of human processes of signification and interpretation in shaping individuals and societies, and focuses on social meaning-making practices of all types, whether visual, verbal or aural in nature, exploring human signifying practices in a specific social and cultural environment, and attempts to explain meaning-making as a social practice to develop an analytical and theoretical framework that can interpret the social context of meaning-making .
\end{abstract}

Key words: social semiotics, related interpretation

\section{INTRODUCTION}

In the two major traditions of semiotics: Saussure's semiotics; and C.S. Peirce's semiotics, Saussure's approach is a generalization of formal, structuralist linguistics; Pearce's theory is an extension of reasoning and logic in the natural sciences. At present, the two schools of semiotics are beginning to merge. On the one hand, many scholars are employing the methods of formal linguistics (especially relational network analysis) to interpret our understanding of culture; on the other hand, they are employing cultural and social information to interpret our understanding of linguistic structures and functions. There have emerged more schools of semiotics, which are of their own characteristics and functions, while social semiotics is one of them.

\section{SOCIAL SEMIOTICS AND THE RELATED INTERPRETATION}

Social semiotics investigates semiotic practices, specific to a culture and community, in order to produce various texts and meanings in various situational contexts and in the contexts of culturally meaningful activities. In Halliday's view, social semiotics should accomplish three tasks: Firstly, to explain the changes, irregularities, dissonance and tension peculiar to human interaction and social process; secondly, to explain the semiotics of social structure, in its aspects both of persistence and of change, including the semantics of social class, of the power system, of hierarchy and of social conflict; thirdly, to explain the linguistic processes in which the members construct social semiotics, in which social reality is shaped, constrained, and modified. Far from tending towards an ideal construction, these processes acknowledge and even institutionalize myopia, prejudice, and misunderstanding [1].

To accomplish these tasks, Hodge and Kress emphasize the importance of the two theoretical premises that underlie social semiotics: firstly, the primacy of the social dimension in understanding language structures and processes; secondly, no single code can be successfully examined or fully understood in isolation [2].

Social semiotics has gone beyond structuralist focus on the internal relationship between the parts of a self-contained system, attempting to explore the use of symbols in particular social situations. Structuralist semioticians pay more attention to the relationship between elements. Social semioticians also stress the importance of the significance attached to the symbols 
in the text by the reader, and are concerned with the system of rules governing the text, emphasizing the role of the semiotic context in producing meaning.

Social semiotics has taken in the core ideas of mainstream semiotics (Saussure; Pierce; Morris) and in the meantime has eliminated the defects that not social functions of the semiotic system, but the structures and codes are stressed; not participants of semiotic activities, but the systems are stressed; not the specific social context, but the products of semiotic activities are stressed. Social semiotics has derived linguistic nutrients from Halliday's theories, rational thoughts of sociology, literature and philosophy from the theories of Marx and Engels, Voloshinov and Lacan,etc. Social semiotics holds that the nature of communication is a type of process rather than a set of disconnected meanings and texts. Meaning is generated in specific social conditions through concrete material forms or media. They are associated with specific subjects and objects, and are interpreted in terms of this connection. The subject and object of texts, contexts and meanings, the social structure and power, and their mutual relations have become the targets of semiotic analysis.

\subsection{CODE, ENCODE AND DECODE}

In semiotics, a code is a set of conventions or sub-codes employed now to convey meanings. The concept of code is primary in semiotics. It is the rule by which given elements are selected and combined to produce new elements. Particular semiotic systems are called codes. The most common is one's written language.

Codes can be divided into structural code and processual code [3]. The structural code is the one by which elements make up a static system and is often referred to as the structure of the system. There is often a distinction between digital code and analog code. Digital codes encode elements in line with absolute differences (either-or-relations), such as between phonemes defined by distinctive features; analog codes organizes elements through progressive approximation (more-or-less relations), for example, words and sentences can be organized into a paragraph, or even a text. The processual code is the rule by which these structures relate to each other. When structural codes for expression elements and for content elements as independent systems interact with processual codes for the relation between expression and content, symbols are produced and meanings occur.

Pierre Guillaud put forward three types of basic codes: logical, aesthetic, and social. Umberto Eco came up with 10 basic codes used to shape images: perceptual code, transmission code, recognition codes, tonal codes, iconic codes, iconographic codes, taste and sensitivity codes, rhetorical codes, stylistic codes, and unconscious codes[4]. The value of any such typology must be clearly assessed in terms of the interpretive light which they shed on the phenomena which they are used to explore.

Codes are rules-driven systems that imply the choice of signifiers and their collocation to communicate the intended meanings most effectively. Interpreting symbols requires familiarity with sets of conventions or codes now employed to convey meaning. Codes are not only communicative conventions, but also processual systems that run the related contracts in a particular domain. The code organizes symbols into a meaningful system of associating signifiers and signifieds.

Codes are the frameworks for interpretation employed by generators and interpreters of the text. In the course of text creation, we choose and integrate symbols associated with the codes we are familiar with to limit the range of the meaning that may arise when absorbed by readers [5]. Codes help make phenomena simple so that experiences can be communicated more easily [6].

In semiotics, the important thing is not the symbol itself, but the set of rules for interpreting the symbol, for it is the life of the semiotic system. Every semiotic system has a set of rules, and these rules become the codes of the semiotic system. Encoding is to employ codes to process or create information transmitted by the sender; while the process of employing codes to interpret the information issued by the sender to the recipient is decoding. Clearly, information can be transmitted correctly only if encoding corresponds to decoding; otherwise there will arise misunderstandings. The sender of the information encodes the meaning, and the recipient decodes it by recalling past experience and placing the information in the proper cultural context.

\subsection{Code and Text}

Roman Jacobson stressed that the generation and interpretation of texts relies on the existence of codes or conventions of communication[7]. Because the meaning of a symbol rests on the code in which it resides, codes supply a framework within which symbols make sense. In fact, if something doesn't work in the code, we cannot give it the status of a symbol. Moreover, if the relation between the signifier and the signified is relatively arbitrary, then it is obvious that the interpretation of the regular meaning of the symbol needs familiarity with the proper set of conventions. Creating the text requires associating it with related codes.

In short, every text is a semiotic system, organized in line with codes and sub-codes, reflecting certain values, 
attitudes, beliefs, assumptions, and practices. It is thought that symbols and codes in a text can relate to each other in some unforeseeable ways, so that semiosis is always open and probably unlimited.

The textual code may be referred as a set of reading ways shared by its generator and reader. The main textual code concerned with the construction of the subject is genre code. Genre is ostensibly neutral, and for those familiar with genre, its function is to make the form (the conventions of the genre) more transparent, highlighting the unique content of individual texts. Genre, of course, supplies a vital frame of reference to help readers identify, choose, and interpret texts, and to help generators work economically in the medium. Nevertheless, a genre can also be considered embodying some values and ideological assumptions, and attempting to form a specific worldview.

When it comes to the concept of genre in the new media age, Kress believes that mixed genres are commonplace. He sees genre as design. A new thinking way may be that, with a general understanding of the scope of genres, of their shapes, and contexts, speakers and writers recently use available resources to create the generic forms[8].

\subsection{MODE OF LOCATING A TEXT}

From the perspective of social semiotics, our understanding of reality cannot be separated from the actual compiled code, semantic system, which means that social (cultural) reality itself is a building full of meanings----a type of semiotic structure. For this reason, such semioticians as Halliday integrate text, genre, code, linguistic system and its social structure into their study of semiotic theories.

Text, in its most general sense, is employed to indicate anything that may be read for meaning; For some scholars, the world is a kind of social text. Text is defined as any phenomenon that conveys information by means of digitally or analogically encoded symbols. Here, communicative behavior is at the core. For most scholars, a text is a system of symbols (in the form of words, images, sounds, and/or gestures). It is constructed and interpreted in line with the conventions related to a genre and in a specific communicative medium. From this perspective, written texts, paintings, and folk costumes are all texts that convey intended and/or traditional information[9].

Semiotics provides us with some conceptual crowbars to construct or deconstruct the codes that work in specific texts and practices, and as long as we can find some gaps, it will give us the opportunity to make an impact.

All communication relies on the application of codes. Jacobson thought that in order for reading to occur, a sender (writer) must issue information to a recipient (reader). For information to be operative, there must be a context (a situation in which the two can be combined), a code shared by both writer and reader, and a contact (a physical channel and psychological contact that keep readers' attention focused on the information). The verbal structure of the information relies mainly on which of these six functions is central. The information focusing on the writer, expression of the writer's emotion itself, is emotive. The information focusing on the reader is conative. The information focusing on the context between reader and writer is referential. Those designed to establish, prolong, or test the validity of the connection between reader and writer are phatic. Those designed to study or examine the commomality of codes in use are metalingual. The self-focused information is poetic. Therefore, all texts are located at some point on a dual axis. The following is Jacobson's formulation of six elements for the communicative behavior [10] :

addresser (writer) $\stackrel{\begin{array}{c}\text { context } \\ \text { (referential) } \\ \text { message } \\ \text { (poetic) }\end{array}}{\stackrel{\text { contact }}{\text { (phatic) }} \begin{array}{l}\text { code } \\ \text { (metalingual) }\end{array}}$

Figure 1 Six-element Formulation of a Communicative Act

The reader who cannot locate the text intuitively will receive garbled information. Information cannot be generated at all by the writer and the reader without a context to relate to each other, without a common code, or without access to physical and psychological contact[11].

Jacobson's mode shows that information and meaning cannot be separated from these constitutive contextual elements. There is an interactive relationship between them. Diverse codes may overlap one another, and the semiotic analysis of any text or practice is concerned with pondering some types of code and the relations between them.

Semiotics is closely connected to the generation of texts, as this activity involves the transmission of information through the use and interpretation of symbols in social communication. The greatest advantage of semiotics compared with other methods of communication between languages is that it is concerned with all types of symbols and codes, particularly with language as the most comprehensive and complex of all the semiotic systems employed by humans[12]. One distinct advantage of the semiotic 
approach to meaning is that it requires equal attention to be paid to designative and associative meaning, since symbols of whatever nature must be understood in the light of the general context of communication and with reference to any and all other symbols combined with linguistic symbols.

Therefore, Social semiotics is to explore signifying practices of humans in a specific social and cultural environment, and attempts to interpret meaning-making as a social practice to develop an analytical and theoretical framework that can expound the social context of meaning-making.

\section{CONCLUSION}

Semiotics admits that semiotic systems are of a conventional feature and that they can be employed to objectively classify the elements filling the reality. Semiotics stresses and focuses on the real related characteristics discovered in system-objects; namely, their existence in reality cannot be separated from the semiotic system used to organize and construct them, and that such features must be related because they help to attach importance to an object, giving it a meaning to determine what it is, rather than describing it as broadly as possible. The semiotic systems themselves belong to the world to which they refer, and the semiotic codes also belong to the world to which they are to organize.

In short, there are certain implications for the socio-semiotic approach, mainly because it is more inclusive. Social semiotics helps us find and explain the processes of semiotic interactions, not only within the text, but also beyond the text. It even expands and broadens the field of study beyond the verbal perspective.

\section{ACKNOWLEDGEMENTS}

I would like to thank Professor Hu Shu zhong, who was kind and gracious, and I often feel that he was strict with me in the process of communicating with him. Starting from the selection of the topic for this paper, he constantly gave me inspiration and direction in choosing the topic and various methods on how to determine the content of my research. Throughout the process of writing and revising, he worked tirelessly to review and helped me clarify my ideas so that this paper could be successfully completed.

\section{REFERENCES}

[1] Halliday, Michael A. K. Language as a Social Semiotic: The Social Interpretation of Language and Meaning. London: Edward Arnold, 1978:126.

[2] Hodge, Robert, and Gunther Kress. Social Semiotics. Ithaca: Cornell UP, 1988: vii-viii.

[3] Johansen, Jørgen Dines, and Svend Erik Larsen. Signs in Use. New York: Routledge, 2002:200.

[4] Guiraud, Pierre. Semiology. Trans. George Gross. London: Routledge and Kegan Paul, 1975:35-38.

[5] Turner, Graeme. British Cultural Studies: An Introduction. New York: Routledge, 1992:17.

[6] Turner, Graeme. British Cultural Studies: An Introduction. New York: Routledge, 1992:35.

[7] Jakobson, Roman. "Language in Relation to Other Communication Systems." Selected Writings, Vol. 2. Ed. Roman Jakobson.Mouton: The Hague, 1971. 570-579.

[8] Kress, Gunther R. Literacy in the New Media Age. New York: Routledge, 2003:121.

[9] Johansen, Jørgen Dines, and Svend Erik Larsen. Signs in Use. New York: Routledge, 2002:220.

[10] Jakobson, Roman. "Closing Statement: Linguistics and Poetics." A Style in Language. Ed. Thomas Sebeok. Cambridge, MA: MIT Press, 1960. 353.

[11] Neel, Jasper. "Reading and Writing." Research in Composition and Rhetoric: A Bibliographic Sourcebook. Ed. Michael G. Moran and Ronald F. Lunsford. Westport/ London: Greenwood Press, 1984: 160 .

[12] Eugene, A. Nida. Language, Culture and Translating. Shanghai: Shanghai Foreign Language Education Press, 1993: 164. 